\title{
Persistência de agrotóxicos utilizados na cultura do milho ao parasitoide Trichogramma pretiosum Riley, 1879 (Hymenoptera: Trichogrammatidae)
}

\author{
Persistence of pesticides used in corn field to the parasitoid Trichogramma pretiosum Riley, 1879 \\ (Hymenoptera: Trichogrammatidae)
}

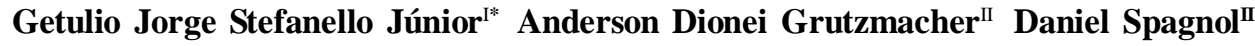 \\ Rafael Antonio Pasini ${ }^{\mathrm{II}}$ Cibele Bonez ${ }^{\mathrm{II}}$ Daiane Carvalho Moreira ${ }^{\mathrm{II}}$
}

\section{RESUMO}

\begin{abstract}
As altas produtividades na cultura do milho têm sido alcançadas em função de vários fatores técnicos, sendo o controle de pragas um dos mais importantes. Nesse sentido, o objetivo deste trabalho foi avaliar a persistência de agrotóxicos utilizados na cultura do milho ao parasitoide Trichogramma pretiosum. Foram avaliadas as persistências de sete inseticidas e três fungicidas, os quais foram diluídos separadamente em $200 L$ de água e pulverizados sobre folhas de videira. Essas folhas foram levadas ao laboratório e utilizadas dentro de gaiolas aos 3, 10, 17, 24 e 31 dias, onde permaneceram confinados os adultos do parasitoide para contaminação. A mortalidade dos adultos de T. pretiosum foi mensurada indiretamente em função do menor número de ovos parasitados do hospedeiro alternativo Anagasta kuehniella. Os resultados foram comparados com o tratamento testemunha $e$ os agrotóxicos classificados conforme categorização da IOBC. Foram utilizadas quatro repetições para cada tratamento. Os fungicidas Opera, Priori Xtra e Folicur 200 EC foram de vida curta (classe 1). Os inseticidas Match EC e Sumithion 500 CE foram de vida curta (classe 1); Lorsban 480 BR e Safety foram moderadamente persistentes (classe 3) e Engeo Pleno, Karate Zeon 50 CS e Tracer foram persistentes (classe 4) aos adultos de T. pretiosum.
\end{abstract}

Palavras-chave: seletividade, parasitoide de ovos, controle biológico, controle químico, toxicidade, agroquímicos, Zea mays.

\section{ABSTRACT}

The high yields in corn have been achieved due to various technical factors, being the pest control one of the most important. In this way, the objective was to evaluate the persistence of the pesticides used in corn field to the parasitoid
Trichogramma pretiosum. It was evaluated the persistence of seven insecticides and three fungicides, which were separately diluted to 200L of water and sprayed on the vine leaves. These leaves were taken to the laboratory and used into cages at 3 , $10,17,24$ and 31 days, where remained confined the adults of the parasitoids to contamination. The adult mortality of $\boldsymbol{T}$. pretiosum was measured according to the lower number of parasitized eggs of alternative host Anagasta kuehniella. The results were compared with the witness treatment and pesticides classified according to IOBC categorization. Four replicates for each treatment were used. The fungicides Opera, Priori Xtra and Folicur 200 EC were short life (class 1). The insecticides Match EC and Sumithion 500 CE were short life (class 1); Lorsban 480 BR and Safety were moderately persistent (class 3), and Engeo Pleno, Karate Zeon 50 CS and Tracer were persistent (class 4) to adults of T. pretiosum.

Key words: side effects, egg parasitoid, biological control, chemical control, toxicity, agrochemicals, Zea mays.

\section{INTRODUÇÃO}

O milho caracteriza-se por ser cultivado em diversas propriedades rurais, sendo utilizado na alimentação animal ou comercializado para posterior processamento. Atualmente, no Brasil, a cultura ocupa cerca de 13,8 milhões de hectares, com produção total de 51,2 milhões de toneladas e produtividade média aproximada de $3.700 \mathrm{~kg} \mathrm{ha}^{-1}$ (IBGE, 2010). Essa produtividade média nacional, entretanto, está aquém do observado em alguns Estados, como no Distrito

\footnotetext{
Instituto Federal de Educação, Ciência e Tecnologia do Rio Grande do Sul, Campus Sertão (IFRS), Sertão, RS, Brasil.

"Departamento de Fitossanidade, Universidade Federal de Pelotas (UFPel), CP 354, 96010-900, Capão do Leão, RS, Brasil. Email: adgrutzm@ufpel.edu.br. Autor para correspondência.
} 
Federal onde já se registrou mais de seis toneladas por hectare (CONAB, 2010). Nesse contexto, é pauta de discussão o manejo da cultura para elevar a produtividade. Ademais, as projeções do agronegócio brasileiro apontam para uma produtividade média esperada de $4.400 \mathrm{~kg} \mathrm{ha}^{-1}$ para a safra 2018/19 (MAPA, 2009).

Nesse cenário, um dos fatores que contribuem para as altas produtividades é o manejo adequado das pragas e doenças que assume grande importância durante as fases vegetativa e reprodutiva da cultura. Atualmente, há mais de 40 espécies de insetos-praga e 25 doenças associadas à cultura do milho no Brasil (AGROFIT, 2010), sendo algumas dessas responsáveis por prejuízos econômicos significativos quando não manejadas adequadamente.

Para isso, o controle químico é o método mais utilizado no manejo fitossanitário de lavouras e, embora eficientes na maioria das vezes, os agrotóxicos podem afetar negativamente a comunidade de inimigos naturais, sobretudo os parasitoides. Dentre esses, está o parasitoide de ovos do gênero Trichogramma que, em função de seu comportamento fototrópico positivo, fica mais exposto ao contato e efeitos negativos dos agrotóxicos (CRUZ, 1995). Além disso, é crescente o uso de agentes de controle biológico em culturas agrícolas do Brasil, já tendo sido registrado o uso de Trichogramma pretiosum Riley, 1879 (Hymenoptera: Trichogrammatidae) em mais de 3.000 hectares de milho em 2008 na Região de Santa Maria, RS (NAVA \& NACHTIGAL, 2010).

Os controles biológico e químico necessitam, então, ser compatibilizados a fim de preservar a comunidade de insetos benéficos no sistema de produção. Nesse sentido, o grupo de trabalho da International Organization for Biological Control of Noxious Animals and Plants (IOBC), West Palaearctic Regional Section (WPRS) estabeleceu as diretrizes para a condução de bioensaios e categorização dos agrotóxicos quanto à seletividade, contando com as etapas de laboratório (fases adulta e imatura); laboratório/casa-de-vegetação (persistência) e campo (fase adulta) (HASSAN et al., 2000; HASSAN \&ABDELGADER, 2001).

No Brasil, entretanto, pesquisas sobre persistência da ação de agrotóxicos sobre T. pretiosum, utilizando a metodologia da IOBC/WPRS, foram realizadas apenas para algumas frutíferas, nas quais foram testados produtos utilizados para a cultura do pessegueiro (GIOLO et al., 2008) e macieira (NÖRNBERG, 2008). Devido à carência de trabalhos de avaliação da persistência de agrotóxicos aplicados na cultura do milho para inimigos naturais, o trabalho objetivou estudar a persistência de efeitos de inseticidas e fungicidas, utilizados na cultura do milho, sobre adultos de $\boldsymbol{T}$. pretiosum.

\section{MATERIAL E MÉTODOS}

Os bioensaios foram conduzidos no Laboratório de Entomologia do Departamento de Fitossanidade, Faculdade de Agronomia "Eliseu Maciel", Universidade Federal de Pelotas, Pelotas, RS, utilizando-se as metodologias estabelecidas pela IOBC/WPRS e descritas por HASSAN et al. (2000) e HASSAN \& ABDELGADER (2001).

Os testes consistiram em pulverizar caldas com agrotóxicos em folhas de videira, as quais foram coletadas em diferentes períodos de tempo para avaliação indireta sobre a mortalidade de adultos de $\boldsymbol{T}$. pretiosum e a classificação quanto à sua persistência. Para essa etapa, foram selecionados os agrotóxicos classificados como nocivos a espécimes adultos do parasitoide (STEFANELLO JÚNIOR, 2007), tais como os inseticidas [grupo químico (máxima dosagem registrada para a cultura do milho em $\mathrm{L} \mathrm{ha}^{-1}$ / concentração de ingrediente ativo na calda, em \%)]: Engeo Pleno [piretróide + neonicotinóide $(0,25 / 0,01325$ lambda-cialotrina $+0,017625$ tiametoxam)]; Karate Zeon 50 CS [piretróide (0,15/0,00375 lambda-cialotrina)]; Lorsban $480 \mathrm{BR}$ [organofosforado $(1,00 / 0,24$ clorpirifós)]; Match EC [benzoiluréia (0,30/0,0075 lufenurom)]; Safety [éter difenílico $(0,10 / 0,015$ etofenproxi)]; Sumithion 500 CE [organofosforado (1,50/ 0,375 fenitrotiona)]; Tracer [espinosinas $(0,10 / 0,024$ espinosade)]; e os fungicidas Folicur 200 EC [triazol $(1,00 / 0,1$ tebuconazol)]; Opera [triazol + estrobilurina $(0,75 / 0,01875$ epoxiconazol $+0,049875$ piraclostrobina $)]$ e Priori Xtra [triazol + estrobilurina $(0,30 / 0,012$ ciproconazol + 0,03 azoxistrobina) ].

As videiras, utilizadas como plantas padrão nas metodologias da IOBC/WPRS, da cultivar Bordô foram adquiridas na forma de mudas enraizadas e, posteriormente, cultivadas em vasos em casa-devegetação. Após o brotamento e emissão de aproximadamente 25 a 30 folhas, as plantas foram submetidas a pulverizações, até o ponto de escorrimento, com agrotóxicos diluídos em 200L de água, utilizando-se pulverizador manual com capacidade de $580 \mathrm{~mL}$. O volume de calda aplicado em cada planta variou em função do número e tamanho das folhas, haja vista que a pulverização tinha o propósito de depositar a calda do agrotóxico sobre as folhas e evitar o seu escorrimento. Posteriormente, as folhas de videira foram coletadas aos 3, 10, 17, 24 e 31 dias e utilizadas na montagem de gaiolas de exposição (HASSAN et al., 2000), onde os adultos de T. pretiosum 
foram colocados em contato com a superfície da folha tratada (HASSAN \& ABDELGADER, 2001).

A população de parasitoides introduzida nas gaiolas, em cada semana do bioensaio, foi oriunda de uma criação mantida sob ovos do hospedeiro Anagasta kuehniella Zeller (1879) (Lepidoptera: Pyralidae) sob condições controladas de temperatura de $25 \pm 2^{\circ} \mathrm{C}$, umidade relativa de $70 \pm 10 \%$ e fotofase de 14 horas. Dessa forma, $300 \pm 50$ adultos de T. pretiosum com até 24 horas de emergência, provenientes de ovos de $\boldsymbol{A}$. kuehniella parasitados e aderidos com goma arábica em forma de círculo de $1 \mathrm{~cm}$ de diâmetro em cartões de papel branco, foram inseridos nas gaiolas por meio de tubos de emergência (ampolas de vidro transparente de $120 \mathrm{~mm}$ de comprimento x $20 \mathrm{~mm}$ de diâmetro em um das extremidades $x 7 \mathrm{~mm}$ na outra), os quais ficaram conectados por 16 horas. A entrada dos parasitoides em cada gaiola foi acelerada por quatro horas, a partir do aumento da intensidade luminosa na sala de testes e, depois da desconexão, os tubos foram mantidos nas mesmas condições da criação por mais três dias, para serem utilizados no cálculo do número de insetos que entraram na gaiola.

A determinação do parasitismo de $\boldsymbol{T}$. pretiosum foi realizada em cinco semanas, correspondendo à coleta das folhas de videira aos 3 , $10,17,24$ e 31 dias após a pulverização. Assim, em cada uma das semanas, foram ofertados cartões contendo três círculos de $1 \mathrm{~cm}$ de diâmetro com $450 \pm 50$ ovos inviabilizados de A. kuehniella, aderidos com goma arábica e alimento, às 24 horas (três cartões), 48 horas (dois cartões) e 96 horas (um cartão) aos adultos de $\boldsymbol{T}$. pretiosum, os quais permaneceram por 144 horas (seis dias) para parasitismo. Sete dias após o início do bioensaio, as gaiolas foram desmontadas, os cartões foram acondicionados em placas de Petri (90x15mm) e mantidos por mais três dias nas mesmas condições da criação, para que os ovos parasitados se tornassem escuros e pudessem ser contabilizados.

O parasitismo de $T$. pretiosum em cada tratamento foi mensurado a partir da contagem dos ovos parasitados em lupa binocular estereoscópica e os dados comparados com a testemunha e, em função da redução do parasitismo, foram classificados em inócuo (classe $1=<30 \%$ ), levemente nocivo (classe $2=$ 31-80\%), moderadamente nocivo (classe $3=81-99 \%$ ) e nocivo (classe $4=>99 \%$ ). De acordo com a IOBC/ WPRS, a classe da persistência de cada agrotóxico foi atribuída quando este causou menos de $30 \%$ de redução no parasitismo, em duas semanas consecutivas e ou ao final do bioensaio. Assim, o agrotóxico foi classificado em: vida curta (classe $1=<5$ dias de atividade tóxica), levemente persistente (classe $2=5$ -
15 dias), moderadamente persistente (classe $3=16-30$ dias) e persistente (classe $4=>31$ dias).

Dessa forma, cada bioensaio realizado para determinar a persistência de determinado agrotóxico a T. pretiosum consistiu em avaliar, indiretamente, o seu efeito sobre populações de adultos do parasitoide durante cinco semanas. Em função da capacidade laboratorial, foram realizados dois bioensaios em que, para cada semana, foram testados seis tratamentos com quatro repetições. Cada repetição foi representada por uma gaiola, que foi considerada uma unidade experimental no delineamento inteiramente casualizado. Análises estatísticas complementares foram realizadas com auxílio do programa estatístico SAS - Statistical Analysis System (SAS LEARNING EDITION, 2002). Os resultados obtidos quanto ao número médio de ovos parasitados por fêmea nos tratamentos, em cada semana, foram testados quanto à normalidade pelo teste Shapiro-Wilk através do procedimento UNIVARIATE. Nos bioensaios em que não se verificou a normalidade dos dados, foi realizada análise nãoparamétrica pelo procedimento NPAR1WAY. Após comprovação da existência de diferença entre tratamentos, os dados foram transformados pelo procedimento RANK e a comparação de médias foi obtida pelo teste de Bonferroni Dunn- $t$, utilizando o procedimento GLM, ao nível de 5\% de probabilidade de erro.

Para comparação dos resultados de persistência, priorizaram-se aqueles realizados que utilizaram a metodologia da IOBC/WPRS, tanto internacionais como nacionais, embora estes tenham utilizado doses registradas para pessegueiro (GIOLO et al., 2008) e macieira (NÖRNBERG, 2008). Da mesma forma, foi priorizada a comparação do produto comercial (HASSAN \& ABDELGADER, 2001) e sua respectiva concentração na calda, pois em alguns agrotóxicos a toxicidade pode ser maior devido à sua formulação e ou ingredientes inertes.

\section{RESULTADOS E DISCUSSÃO}

De acordo com os resultados de parasitismo de T. pretiosum no presente trabalho, verificou-se que na testemunha do bioensaio 1 o parasitismo variou de 19,22 a 35,54 ovos por fêmea e de 17,92 a 45,28 no bioensaio 2 (Tabelas 1 e 2), durante as cinco semanas do teste, estando, portanto, acima dos valores mínimos estabelecidos pela IOBC/WPRS.

De acordo com a IOBC/WPRS, um dos critérios de validação dos bioensaios é o número de ovos parasitados por fêmea no tratamento testemunha, haja vista que os resultados dos demais tratamentos 
Tabela 1 - Parasitismo e redução no parasitismo de Trichogramma pretiosum e persistência de agrotóxicos utilizados na cultura do milho, conforme normas da IOBC/WPRS. Pelotas, RS.

\begin{tabular}{|c|c|c|c|c|c|c|}
\hline \multicolumn{7}{|c|}{ Bioensaio 1} \\
\hline \multirow{4}{*}{$\begin{array}{l}\text { Tratamento/Dosage } \\
\mathrm{m}\left(\mathrm{L} \mathrm{ha}^{-1}\right)\end{array}$} & ---------------- & ----------------1 & oós a pulveriz: & - & -------------- & $\mathrm{P}^{3}$ \\
\hline & 3 & 10 & 17 & 24 & 31 & $\mathrm{P}$ \\
\hline & Ovos/fêmea ${ }^{1}$ & Ovos/fêmea & Ovos/fêmea & Ovos/fêmea & Ovos/fêmea & Dias \\
\hline & $\mathrm{RP}(\mathrm{C})^{2}$ & $\mathrm{RP}(\mathrm{C})$ & $\mathrm{RP}(\mathrm{C})$ & $\mathrm{RP}(\mathrm{C})$ & $\mathrm{RP}(\mathrm{C})$ & $(C)^{3}$ \\
\hline Testemunha & $28,77 \mathrm{a}$ & $24,60 a b$ & $35,54 \mathrm{a}$ & $19,22 \mathrm{a}$ & $29,52 \mathrm{a}$ & - \\
\hline \multirow{2}{*}{ Opera/0,75 } & $24,29 \mathrm{ab}$ & $28,51 \mathrm{a}$ & $31,00 \mathrm{a}$ & $20,88 \mathrm{a}$ & $31,83 \mathrm{a}$ & $<5$ \\
\hline & $15,57(1)$ & $0(1)$ & $12,77(1)$ & $0(1)$ & $0(1)$ & (1) \\
\hline \multirow{2}{*}{ Priori Xtra/0,30 } & $26,13 \mathrm{a}$ & $24,70 \mathrm{ab}$ & $32,00 \mathrm{a}$ & $19,96 \mathrm{a}$ & $25,27 \mathrm{ab}$ & $<5$ \\
\hline & $9,18(1)$ & $0(1)$ & $9,96(1)$ & $0(1)$ & $14,40(1)$ & (1) \\
\hline \multirow{2}{*}{$\begin{array}{l}\text { Sumithion } 500 \\
\text { CE/1,50 }\end{array}$} & $0 \mathrm{~d}$ & $19,70 \mathrm{ab}$ & $28,41 \mathrm{ab}$ & $17,91 \mathrm{ab}$ & $30,33 \mathrm{a}$ & $5-15$ \\
\hline & $100(4)$ & $19,92(1)$ & $20,06(1)$ & $6,82(1)$ & $0(1)$ & (1) \\
\hline \multirow{2}{*}{ Safety/0,10 } & $2,82 \mathrm{bc}$ & $9,70 \mathrm{bc}$ & $12,70 \mathrm{bc}$ & $11,41 \mathrm{ab}$ & $26,54 \mathrm{ab}$ & $>30$ \\
\hline & $90,20(3)$ & 60,57 (2) & $64,27(2)$ & $40,63(2)$ & $10,09(1)$ & (3) \\
\hline \multirow{2}{*}{ Tracer/0,10 } & $0,14 \mathrm{~cd}$ & $0,41 \mathrm{c}$ & $0,10 \mathrm{c}$ & $0,49 \mathrm{~b}$ & $0,64 \mathrm{~b}$ & $>30$ \\
\hline & $99,51(4)$ & $98,33(3)$ & $99,72(4)$ & 97,45 (3) & $97,83(3)$ & (4) \\
\hline
\end{tabular}

${ }^{1}$ Médias seguidas por letras idênticas na coluna não diferem estatisticamente $(\mathrm{P}>0,05)$ pelo teste (Kruskal-Wallis) Bonferroni-Dunn $t$.

${ }^{2}$ Redução no parasitismo $(\mathrm{RP})$ em relação à testemunha e classes $(\mathrm{C})$ : 1 = inócuo $(<30 \%) ; 2=1$ evemente nocivo $(30-79 \%)$; 3 = moderadamente nocivo $(80-99 \%) ; 4$ = nocivo $(>99 \%)$.

${ }^{3}$ Persistência $(\mathrm{P})$ em função da duração da atividade tóxica menor que $30 \%$ na redução do parasitismo em dias e classes $(\mathrm{C})$ : $1=$ vida curta (<5 dias); 2 = levemente persistente (5-15 dias); $3=$ moderadamente persistente $(16-30$ dias $)$ e $4=$ persistente $(>30$ dias $)$.

serão comparados com o tratamento testemunha (HASSAN et al., 2000; HASSAN \& ABDELGADER, 2001). De acordo com os referidos autores, esse valor é de no mínimo 15 ovos parasitados por fêmea para a espécie Trichogramma cacoeciae Marchal, 1927 (Hymenoptera: Trichogrammatidae), e, embora esse valor ainda não tenha sido normatizado para $\boldsymbol{T}$. pretiosum, é também utilizado como um critério laboratorial.

Nos bioensaios 1 e 2 , foi verificada a inocuidade dos fungicidas a $\boldsymbol{T}$. pretiosum desde a primeira semana, sendo observados parasitismos similares aos das testemunhas. Dentre eles, Folicur 200 EC foi o que apresentou maior toxicidade ao parasitoide (27,16\%), embora sempre tenha sido classificado como inócuo (classe 1). Quanto à persistência, os fungicidas Opera, Priori Xtra e Folicur 200 EC foram de vida curta, apresentando resíduos tóxicos inferiores a 5 dias, conforme classificação da IOBC/WPRS (Tabelas 1 e 2), pois o efeito nocivo de cada tratamento foi inferior a $30 \%$ quanto à redução no parasitismo desde a primeira semana. Para os inseticidas, foi verificada inocuidade ao parasitoide apenas para o regulador de crescimento Match EC, que foi inócuo desde os 3 dias após a pulverização, e, apesar da redução no parasitismo de ovos próximo a $34 \%$ (classe 2) na última semana avaliada (Tabela 2), foi classificado como de vida curta ( $<5$ dias de toxicidade).
A inocuidade também foi verificada para o inseticida Sumithion 500 CE após os 10 dias após da aplicação (DAP), em testes isolados, e foi categorizado como de vida curta (classe 1) (Tabela 1). Para Lorsban $480 \mathrm{BR}$, a inocuidade foi verificada somente após os 24DAP, em testes isolados, sendo classificado como moderadamente persistente (classe 3) (Tabela 2). Da mesma forma, Safety foi inócuo aos 31DAP, em testes isolados, porém foi classificado como moderadamente persistente (classe 3) quanto à persistência. Os inseticidas Tracer, Karate Zeon 50 CS e Engeo Pleno ainda mantiveram ação tóxica sobre $T$. pretiosum até 31DAP, mensurados pela redução no parasitismo acima de $30 \%$, e foram classificados como persistentes (classe 4) (Tabelas 1 e 2).

De uma maneira geral, os agrotóxicos tenderam a diminuir os efeitos tóxicos e a mortalidade de $\boldsymbol{T}$. pretiosum ao longo das cinco semanas da montagem dos bioensaios. Esse efeito, entretanto, não foi verificado para todos os agrotóxicos avaliados e isto pode estar atribuído às variações de temperatura e umidade observados no interior da casa-de-vegetação, causando a diminuição desuniforme da toxicidade dos produtos aos adultos do parasitoide. Sugere-se, dessa forma, que os bioensaios sejam conduzidos em câmaras climáticas e ou casa-de-vegetação com controle automatizado das condições climáticas.

Ciência Rural, v.42, n.1, jan, 2012. 
Tabela 2 - Parasitismo e redução no parasitismo de Trichogramma pretiosum e persistência de agrotóxicos utilizados na cultura do milho, conforme normas da IOBC/WPRS. Pelotas, RS.

\begin{tabular}{|c|c|c|c|c|c|c|}
\hline \multicolumn{7}{|c|}{ Bioensaio 2} \\
\hline \multirow{4}{*}{$\begin{array}{l}\text { Tratamento/Dosage } \\
\mathrm{m}\left(\mathrm{L} \mathrm{ha}^{-1}\right)\end{array}$} & \multicolumn{5}{|c|}{-----------------------------------------Dias após a pulverização----------------------------------------- } & \multirow{4}{*}{$\begin{array}{c}\mathrm{P}^{3} \\
\text { Dias } \\
(\mathrm{C})^{3}\end{array}$} \\
\hline & 3 & 10 & 17 & 24 & 31 & \\
\hline & Ovos/fêmea ${ }^{1}$ & Ovos/fêmea & Ovos/fêmea & Ovos/fêmea & Ovos/fêmea & \\
\hline & $\mathrm{RP}(\mathrm{C})^{2}$ & $\mathrm{RP}(\mathrm{C})$ & $\mathrm{RP}(\mathrm{C})$ & $\mathrm{RP}(\mathrm{C})$ & $\mathrm{RP}(\mathrm{C})$ & \\
\hline Testemunha & $28,35 \mathrm{ab}$ & $18,26 \mathrm{bc}$ & $17,92 \mathrm{a}$ & 45,28 a & $30,38 \mathrm{a}$ & - \\
\hline \multirow{2}{*}{ Match EC/0,30 } & 27,35 a & 26,05 a & $15,93 \mathrm{ab}$ & $32,03 \mathrm{abc}$ & $19,95 \mathrm{bc}$ & $<5$ \\
\hline & $3,53(1)$ & $0(1)$ & $11,10(1)$ & $29,26(1)$ & $34,33(2)$ & (1) \\
\hline \multirow{2}{*}{ Folicur $200 \mathrm{EC} / 1,00$} & $28,97 \mathrm{ab}$ & $22,95 a b$ & $16,60 \mathrm{ab}$ & $40,32 \mathrm{ab}$ & $22,13 \mathrm{ab}$ & $<5$ \\
\hline & $0(1)$ & $0(1)$ & $7,37(1)$ & $10,95(1)$ & $27,16(1)$ & (1) \\
\hline \multirow{2}{*}{$\begin{array}{l}\text { Lorsban } 480 \\
\text { BR/1,00 }\end{array}$} & $0 \mathrm{c}$ & $1,38 \mathrm{~cd}$ & $9,03 \mathrm{bc}$ & 32,26 abcd & $21,34 \mathrm{~b}$ & $16-30$ \\
\hline & $100(4)$ & $92,44(3)$ & 49,61 (2) & $28,75(1)$ & $29,76(1)$ & (3) \\
\hline \multirow{2}{*}{$\begin{array}{l}\text { Karate Zeon } 50 \\
\text { CS/0,15 }\end{array}$} & $1,02 \mathrm{c}$ & $0,23 \mathrm{de}$ & $5,96 \mathrm{c}$ & 1,97 cde & $8,97 \mathrm{~cd}$ & $>30$ \\
\hline & $96,40(3)$ & $98,74(3)$ & $66,74(2)$ & $95,65(3)$ & $70,47(2)$ & (4) \\
\hline \multirow{2}{*}{ Engeo Pleno/0,25 } & 0 & $0 \mathrm{e}$ & $0 \mathrm{c}$ & $0,04 \mathrm{e}$ & $0,03 \mathrm{~d}$ & $>30$ \\
\hline & $100(4)$ & $100(4)$ & $100(4)$ & 99,91 (4) & $99,90(4)$ & (4) \\
\hline
\end{tabular}

${ }^{1}$ Médias seguidas por letras idênticas na coluna não diferem estatisticamente $(\mathrm{P}>0,05)$ pelo teste (Kruskal-Wallis) Bonferroni-Dunn $t$.

${ }^{2}$ Redução no parasitismo (RP) em relação à testemunha e classes $(\mathrm{C}): 1=$ inócuo $(<30 \%) ; 2=$ levemente nocivo $(30-79 \%) ; 3=$ moderadamente nocivo (80-99\%) e $4=$ nocivo ( $>99 \%$ );

${ }^{3}$ Persistência $(\mathrm{P})$ em função da duração da atividade tóxica menor que $30 \%$ na redução do parasitismo em dias e classes $(\mathrm{C}): 1=$ vida curta ( $<5$ dias); 2 = levemente persistente (5-15 dias); 3 = moderadamente persistente (16-30 dias) e $4=$ persistente ( $>30$ dias).

De acordo com a análise estatística, verificou-se que, para a maioria dos tratamentos dos bioensaios 1 e 2 (Tabelas 1 e 2), a testemunha não diferiu estatisticamente dos agrotóxicos considerados inócuos (classe 1), tais como no bioensaio 1 para Opera, Priori Xtra aos 3DAP; Opera, Priori Xtra e Sumithion $500 \mathrm{CE}$ aos 10, 17 e 24DAP, e Opera, Priori Xtra, Sumithion 500 CE e Safety aos 31DAP; e no bioensaio 2 para Match EC aos 3, 17 e 24DAP, Folicur 200 EC aos 3, 10, 17, 24 e 31DAP, e Lorsban 480 BR aos 24DAP.

Resultados similares para Folicur 250 EC (0,0625\% i.a.) foram observados por STERK et al. (1999), que também classificaram o fungicida como classe 1 (vida curta), embora divirja da concentração utilizada no presente trabalho $(0,1 \%)$. HASSAN et al. (1988) e HASSAN (1994) também verificaram que o fungicida Tilt, nas concentrações de $0,02 \%$ e $0,08 \%$ de ingrediente ativo propiconazole na calda, apresentou vida curta para T. cacoeciae. Resultados de persistência de fungicidas do grupo químico estrobilurina e triazol/ estrobilurinas sobre $\boldsymbol{T}$. pretiosum não foram encontrados na literatura.

HASSAN (1994) encontrou resultados similares para Karate (lambda-cialotrina, 0,075\% i.a.), classificando-o como persistente para T. cacoeciae, embora tenha utilizado como fator de classificação de persistência a redução de $50 \%$ no número de ovos parasitados e concentração duas vezes superior ao do presente trabalho. O mesmo autor classificou o ingrediente ativo clorpirifós $(0,24 \%$ i.a. $)$, marca comercial Dursban Spritzp., como persistente (classe 4) para T. cacoeciae, embora para T. pretiosum o mesmo ingrediente ativo $(0,24 \%$ i.a.), na marca comercial Lorsban $480 \mathrm{BR}$, tenha sido classificado como moderadamente persistente. NÖRNBERG (2008) também verificou efeitos nocivos de Lorsban 480 BR para T. pretiosum até a terceira semana (17 dias após a pulverização) e classificou o inseticida como moderadamente persistente (classe 3 ), embora o autor tenha usado calda com apenas $0,072 \%$ de ingrediente ativo, sendo que no presente trabalho foi testado a $0,24 \%$. Apesar de não terem sido encontrados resultados na literatura para tiametoxam, a elevada toxicidade pode ser devido ao ingrediente ativo lambdacialotrina na maior concentração em Engeo Pleno $(0,01325 \%$ de i.a. na calda), quando comparado com o seu uso isolado em Karate Zeon 50 CS $(0,00375 \%)$, ou ingredientes inertes presentes na formulação comercial.

Para Sumithion 500 CE, classificado como de vida curta (classe 1) no presente trabalho (Tabela 1), GIOLO et al. (2008) o categorizaram comolevemente nocivo (classe 2) a $\boldsymbol{T}$. pretiosum, embora tenham utilizado dosagem cinco vezes inferior, numa concentração de 0,075\% de ingrediente ativo. Embora ainda não confirmado, isso pode revelar uma tendência não persistente do produto, mesmo sendo do mesmo grupo químico de Lorsban $480 \mathrm{BR}$. 
Resultados de persistência baseados na metodologia da IOBC/WPRS não foram encontrados para os demais inseticidas. No entanto, CARVALHO et al. (1999) também observaram que o ingrediente ativo lambda-cialotrina $\left(0,025 \mathrm{~g}\right.$ i.a. $\left.\mathrm{L}^{-1}\right)$ foi persistente para duas linhagens de $\boldsymbol{T}$. pretiosum quando testado sobre plantas de tomateiro, embora tenham utilizado concentração inferior à do presente trabalho $(0,0375 \mathrm{~g}$ i.a. $\left.\mathrm{L}^{-1}\right)$. A persistência de lufenurom $\left(0,4 \mathrm{~g}\right.$ i.a. $\left.\mathrm{L}^{-1}\right)$ também foi avaliada para adultos de T. pretiosum e o agrotóxico foi classificado como levemente nocivo (classe 2) por CARVALHO et al. (2002), análise esta que foi realizada sobre plantas de tomateiro e em concentração maior do que a usada neste trabalho $\left(0,075\right.$ g i.a. $\left.\mathrm{L}^{-1}\right)$. Para lufenurom, CAÑETE (2005) verificou que esse ingrediente ativo $\left(7,5 \mathrm{~g}\right.$ i.a. $\left.\mathrm{L}^{-1}\right)$ apresentou efeito tóxico para os primeiros sete dias, apresentando sobrevivência de T. pretiosum superior a 75\% após esse período. Entretanto, espinosade (12g i.a. ha ${ }^{-1}$ ) e clorpirifós (120g i.a. ha ${ }^{-1}$ ) causaram mortalidade por mais de 100 dias. Nesse caso, no entanto, a persistência do inseticida foi avaliada sobre a superfície interna de tubos de vidro ao longo do tempo, metodologia distinta daquela preconizada pela IOBC/WPRS.

Para espinosade, NASREEN (2003) encontrou redução de parasitismo de 14,28; 31,81 e $35,48 \%$ aos $1 \div$, $2^{\circ}$ e 3 언 dias após a pulverização em plantas de algodoeiro, respectivamente, para Trichogramma chilonis Ishii, 1941 (Hymenoptera: Trichogrammatidae), embora divergentes em espécie de parasitoide e concentração do ingrediente ativo $(28,8 \mathrm{ppm})$ quando comparado à do presente trabalho (240ppm). Resultados quanto à persistência de Safety a $\boldsymbol{T}$. pretiosum não foram encontrados na literatura.

Segundo a metodologia da IOBC/WPRS, os agrotóxicos classificados como de vida curta (classe 1) e levemente persistente (classe 2) são seletivos aos adultos de $\boldsymbol{T}$. pretiosum. No entanto, aqueles classificados como moderadamente persistentes (classe 3) e persistentes (classe 4) necessitam passar para a fase de campo, em lavouras de milho.

\section{CONCLUSÃO}

Os fungicidas Opera ( 0,75$)$, Priori Xtra $(0,30)$ e Folicur 200 EC (1) são de vida curta (classe 1). Os inseticidas Match CE $(0,30)$ e Sumithion 500 CE $(1,50)$ são de vida curta (classe 1); Lorsban 480 BR (1) e Safety $(0,10)$ são moderadamente persistentes (classe 3$) \mathrm{e}$ Engeo Pleno $(0,25)$, Karate Zeon 50 CS $(0,15)$ e Tracer $(0,10)$ são persistentes (classe 4) a adultos de $\boldsymbol{T}$. pretiosum.

\section{REFERÊNCIAS}

AGROFIT: Sistema de agrotóxicos fitossanitários. Disponível em: <http://agrofit.agricultura.gov.br/agrofit_cons/ principal_agrofit_cons>. Online. Acesso em: 10 jul. 2010.

CAÑETE, C.L. Seletividade de inseticidas a espécies de Trichogramma (Hymenoptera: Trichogrammatidae). 2005. 106f. Tese (Doutorado em Ciências - Zoologia) - Curso de Pósgraduação em Zoologia, Universidade Federal do Paraná, PR.

CARVALHO, G.A. et al. Ação residual de alguns inseticidas pulverizados em plantas de tomateiro sobre duas linhagens de Trichogramma pretiosum Riley, 1879 (Hymenoptera: Trichogrammatidae) em casa-de-vegetação. Ciência e Agrotecnologia, v.23, n.4, p.770-775, 1999. Disponível em: <http://www.editora.ufla.br/site/_adm/upload/revista/23-41999_01.pdf>. Acesso em: 21 jul. 2010.

CARVALHO, G.A. et al. Efeitos de alguns inseticidas utilizados na cultura do tomateiro (Lycopersicon esculentum) a Trichogramma pretiosum Riley, 1879 (Hymenoptera: Trichogrammatidae). Ciência e Agrotecnologia, v.26, n.6, p.1160-1166, 2002. Disponível em: <http://www.editora.ufla.br/ site/_adm/upload/revista/26-6-2002_06.pdf >. Acesso em: 18 jul. 2010 .

CONAB. Safras - grãos. Disponível em: <http:// www.conab.gov.br/conabweb/download/safra/ MilhoTotalSerieHist.xls>. Online. Acesso em: 19 jul. 2010.

CRUZ, I. A lagarta-do-cartucho na cultura do milho. Sete Lagoas: Embrapa/Centro Nacional de Pesquisa de Milho e Sorgo, 1995. 45p. (Circular Técnica, 21).

GIOLO, F.P. et al. Persistência de agrotóxicos indicados na produção integrada de pêssego a Trichogramma pretiosum Riley, 1879 (Hymenoptera: Trichogrammatidae). Revista Brasileira de Fruticultura, v.30, n.1, p.122-126, 2008. Disponível em: <http://www.scielo.br/scielo.php?pid=S0100$29452008000100023 \&$ script=sci_arttext $>$. Acesso em: 15 jul. 2010. doi: 10.1590/S0100-29452008000100023.

HASSAN, S.A. Comparison of three different laboratory and one semi-field test methods to asses the side effects of pesticides on Trichogramma cacoeciae. IOBC/WPRS Bulletin, v.17, n.10, p.133-141, 1994.

HASSAN, S.A.; ABDELGADER, H. A sequencial testing program to assess the effects of pesticides on Trichogramma cacoeciae Marchal (Hym., Trichogrammatidae). IOBC/WPRS Bulletin, v.24, n.4, p.71-81, 2001.

HASSAN, S.A. et al. Results of the fourth joint pesticide testing programme carried out by the IOBC/WPRS - Working Group "Pesticides and Beneficial Organisms". Zeitschrift für Angewandte Entomologie, v.105, p.321-329, 1988.

HASSAN, S.A. et al. A laboratory method to evaluate the side effects of plant protection products on Trichogramma cacoeciae Marchal (Hym., Trichogrammatidae). In: CANDOLFI, M.P. et al. (Eds.). Guidelines to evaluate sideeffects of plant protection products to non-target arthropods. Reinheim: IOBC/WPRS. 2000. p.107-119. 
IBGE. Confronto das Safras de 2009 e das Estimativas para 2010 - Brasil. Disponível em: <http://www.ibge.gov.br/ home/estatistica/indicadores/agropecuaria/lspa/ defaulttab.shtm>. Online. Acesso em: 19 jul. 2010.

MAPA. Projeções do Agronegócio: Brasil 2008/09 a 2018/ 19. Brasília: MAPA, 2009. 64p.

NASREEN, A. Selectivity between chemical and biological agents in controlling Helicoverpa armigera (Hubner) in cotton. 2003. 153f. Thesis (PhD) - Faculty of Agriculture, University of Agriculture.

NAVA, D.E.; NACHTIGAL, G.F. Controle biológico no Sul do Brasil. In: G.BIO - Revista de Controle Biológico. Piracicaba: Facile, 2010. p.15-18.

NÖRNBERG, S. Efeito de agrotóxicos utilizados na produção integrada de maçã sobre Trichogramma pretiosum 1879 Riley
Trichogrammatidae). 2008. 76f. Dissertação (Mestrado em Fitossanidade - Entomologia) - Curso de Pós-graduação em Fitossanidade, Universidade Federal de Pelotas, RS.

STEFANELLO JÚNIOR, G.J. Seletividade de agrotóxicos registrados para a cultura do milho a adultos de Trichogramma pretiosum Riley, 1879 (Hymenoptera: Trichogrammatidae) em laboratório. 2007. 75f. Dissertação (Mestrado em Fitossanidade - Entomologia) - Curso de Pós-graduação em Fitossanidade, Universidade Federal de Pelotas, RS

SAS LEARNING EDITION. Getting Started with the SAS Learning Edition. Cary, North Carolina: SAS Institute, 2002. 2 Cd-rom. Statistical Analysis System.

STERK, G. et al. Results of the seventh joint pesticide testing programme carried out by the IOBC/WPRS - Working Group "Pesticides and Beneficial Organisms". Biocontrol, v.44, n.1, p.99-117, 1999. 